\title{
Sleep status and the associated factors, a large cross-sectional study in Shaanxi province of China
}

Yaxuan Zhang

Fudan University

Jiwei Wang

Fudan University

Xinyuan Lu

Fudan University

Beibei Che

Fudan University

\section{Rui Wang}

Health Publicity and Education Center of Shaanxi province, China

Jinming Yu ( $\nabla$ jinmingyu163@163.com )

Fudan University

\section{Research article}

Keywords: Sleep quality, Associated factors, Logistic regression analysis

Posted Date: September 14th, 2020

DOI: https://doi.org/10.21203/rs.3.rs-55325/v1

License: (c) (i) This work is licensed under a Creative Commons Attribution 4.0 International License. Read Full License

Version of Record: A version of this preprint was published at International Journal of Environmental Research and Public Health on January 30th, 2021. See the published version at https://doi.org/10.3390/ijerph18031250. 


\section{Abstract \\ Background}

This study aimed at investigating the sleep status and its associated factors in Shaanxi province of China.

\section{Methods}

This cross-sectional study was conducted among 11399 subjects in Shaanxi Province, China. Data was collected via spot field questionnaire survey. The contents included demographic characteristics, sleep status, lifestyles, disease history and other associated factors. Logistic regression analysis was used to estimate the effect of associated factors for sleep quality. Crude odds ratio (cOR) and adjusted odds ratio (aOR) and their $95 \%$ confident intervals $(\mathrm{Cl})$ were calculated.

\section{Results}

A total of 11036 subjects were included in the final analysis. There were $12.8 \%$ participants having a bad or very bad sleep. In the last month, $8.4 \%$ participants had difficulty in initiating sleep, $7.6 \%$ participants had difficulty in maintaining sleep, $8.8 \%$ participants suffered from awakening earlier and $10.3 \%$ participants had the problem of feeling sleepy during the day $\geq 3$ times per week. There were $16.5 \%$ participants having one of the above sleep problems (difficulty in initiating sleep, difficulty in maintaining sleep, awakening early) $>3$ months. Poorer sleep quality was associated with female (aOR $=1.792, p<$ $0.001)$, unmarried or without cohabiting with boyfriend/girlfriend ( $\mathrm{aOR}=1.319,95 \% \mathrm{Cl}=1.022-1.704)$, divorced or widowed $(a O R=1.436,95 \% \mathrm{Cl}=1.052-1.960)$, heart diseases $(a O R=1.478, p=0.002)$, musculoskeletal diseases $(a O R=1.460, p=0.018)$, concerning about their own health $(a O R=1.113, p=$ $0.016)$, alcohol drinking $(a O R=1.201, p<0.001)$, taking hypnotics $(a O R=1.643, p<0.001)$, a longer daily screen time $(a O R=1.055, p<0.001)$. Better sleep quality was associated with medium education level $(a O R=0.748, p=0.003)$, high family monthly income $(a O R=0.939, p=0.004)$, good self-reported health status $(a O R=0.490, p<0.001)$, having breakfast regularly $(a O R=0.935, p=0.016)$.

\section{Conclusions}

The self-reported sleep quality was generally good in Shaanxi, China. Sleep quality was associated with gender, marital status, educational level, family monthly income, heart disease, musculoskeletal diseases, degree of concerning about their own health, self-reported health status, alcohol drinking, having breakfast, take hypnotics and daily screen time.

\section{Background}


Sleep disturbance is becoming a more and more important health issue around the world. Sleep disorders may influence people's daily life and give rise to many health problems. Sleep deprivation will affect human's physical and cognitive performance[1]. Sleep apnea may cause a batch of cardiovascular consequences, such as hypertension, coronary heart disease, arrhythmia, heart failure, and stroke[2]. Lack of sleep may have a negative impact on weight management [3]. Impaired sleep is both a risk factor and a symptom of depression [4]. Appropriate sleep duration has a protective effect on type 2 diabetes compared with short or long sleep duration [5].

With the development of China, people face fierce competition and suffer from great life stress, which increases people's anxiety. Anxiety symptoms are associated with sleep problems [6]. Nevertheless, few studies have focused on people's sleep quality and its associated factors in China.

Several previous studies investigated sleep quality of students [7, 8]. A cross-sectional study among students in Macao of China showed that irregular bedtime was associated with elevated body weight [7]. Another cross-sectional survey among Inner Mongolia Medical University students found that exercise less than three times a week and skipping breakfast were associated with poor sleep quality [8]. Some research investigated sleep quality and its associated factors among rural adults $[9,10]$, low-income rural adults [11], rural elderly $[12,13]$. A cross-sectional survey conducted in Deqing County of China reported that rural people who were unmarried or had low personal income or any chronic diseases were more likely to have poor sleep quality [9]. Another study among rural adults in Henan province of China showed that older age, being female, unmarried/divorced/widowed, low education level, low income, drinking, depression and dyslipidemia could increase the odds of poor sleep quality [10]. A cross-sectional survey among low-income adults in a rural area of China found that older age, unemployment, lower income, disability and chronic disease comorbidities were associated with poor sleep quality [11]. Two crosssectional studies among rural elderly in China showed that cardiometabolic risk factors and disorders were associated with poor sleep quality [12], chronic disease, advanced age, low quantities of staple food, rice as major food, poor Physical Component Summary, poor Mental Component Summary, and significant dysfunction of Activities of Daily Living were predictors for poor sleep quality [13].

In this case, our research investigated sleep quality and its associated factors among adults from urban and rural areas on a large scale in Shaanxi Province, China.

\section{Methods}

\section{Study site and population}

This cross-sectional study was conducted in Shaanxi Province, China. Seven cities and one demonstration zone were involved in this study. They were Yan'an city, Yulin city, Ankang city, Hanzhong city, Xi'an city, Weinan city, Baoji city and Yangling Demonstration Zone.

\section{Investigation methods}


We extracted one district and one county from each city, which ensured that urban population and rural population were both investigated. Subjects were convenient sampled as clusters from urban or rural community in May to June 2019. Participants which aged from 18 to 90 and could read and complete the questionnaire were recruited. Spot field questionnaire survey were conducted by trained investigators. Data were collected anonymously.

The study was conducted in accordance with the Helsinki declaration and approved by the Ethics Committee for Medical Research, School of Public Health, Fudan University.

Written informed consent was obtained from all participants or their legal guardians before investigation. The questionnaire used in our study was developed for this study. An additional file shows the English language version of the questionnaire in more detail [see Additional file 1].

The questionnaire contained sociodemographic characteristics, lifestyles, health status, disease history and self-reported sleep quality.

Sociodemographic characteristics include sex, age, marital status, educational level, personal monthly income, and family monthly income. Body mass index (BMI) is calculated by weight(kg)/ height ${ }^{2}\left(\mathrm{~m}^{2}\right)$ and categorized into 4 groups [14].

Lifestyle factors include physical activities, smoking, alcohol drinking, having breakfast, eating vegetables, eating fruits, square dancing, daily screen time, using electronic devices before sleep, time of exposure to sunlight. Physical activities is measured by average times of at least 30 minutes of physical activity (physical activity of moderate intensity or above, such as brisk walking, running, swimming, dancing, cardio, football, basketball, climbing, etc.) per week. Smoking is measured by average number of cigarettes smoked each day. Alcohol drinking is measured by frequency of drinking. Having breakfast is measured by average times of having breakfast per week. Eating vegetables is measured by average weight of vegetables you have each day which excluded starchy vegetables such as potato, yam, taro, etc. Eating fruits is measured by average times of eating fresh fruits per week which excluded canned fruit, preserved fruit, etc. Square dancing is defined as clustered dance in outdoor places such as parks in China and measured by average times per week. Daily screen time is measured by average time of daily use of electronic devices such as cellphone, television and computers. Using electronic devices before sleep is defined as using electronic devices between bedtime and 30 minutes before bedtime, and measured by how often. Time of exposure to sunlight is measured by daily average sunlight exposure time (minutes) in the last month.

Subjects were asked the degree of concerning about their own health and self-reported health status.

We also measured whether they were suffering from chronic noncommunicable diseases which were diagnosed by a doctor. Chronic diseases in our study included hypertension, diabetes, hyperlipidemia, heart disease (such as coronary heart disease, rheumatic heart disease, cardiomyopathy, congenital heart disease, pulmonary heart disease and so on), respiratory diseases (such as impaired lung function, 
chronic obstructive pulmonary disease, asthma and so on), stroke (such as transient ischemic attack, ischemic stroke, hemorrhagic stroke and so on), malignant tumor, hyperuricemia, musculoskeletal diseases (such as osteoarthritis, rheumatoid arthritis and so on).

\section{Measurement of sleep quality}

Self-reported sleep quality (hereinafter referred to as sleep quality) was used with a 5-ordinal-categorized answer, which means that answer 1 to 5 correspond with very good, good, general, bad and very bad, respectively.

According to diagnosis of insomnia in the Diagnostic and Statistical Manual of Mental Disorders, Fifth Edition (DSM-V)[15], Participants were asked the following questions to get more sleep information:

1. In the last month, the frequency of difficulty initiating sleep for at least 30 minutes;

2. In the last month, the frequency of difficulty maintaining sleep, waking more than 3 times in a night;

3. In the last month, the frequency of awakening earlier than usual in the morning, at least 30 minutes early, with inability to return to sleep;

4. In the last month, the frequency of feeling sleepy during the day;

5. How long have you had one of above sleep problems (difficulty initiating sleep, difficulty maintaining sleep, awakening early)?

6. In the last month, have you take medication (e.hypnotics such as benzodiazepines or to help you sleep?

\section{Statistical analysis}

Data entry was completed by Epidata 3.1 and statistical analysis was performed by SAS 9.4 (Copyright by SAS Institute Inc., Cary, NC. Licensed to FUDAN UNIVERSITY). The significance level was set at 0.05 . Continuous variables were described as mean and standard deviation (SD), and categorical variables were described as frequencies and percentage (\%).

Logistic regression analysis was used to estimate the effect of influencing factors on sleep quality, crude odds ratio (cOR) and adjust odds ratio (aOR) and their $95 \%$ confident intervals $(\mathrm{Cl})$ were calculated. Selfreported sleep quality was categorized as good sleep quality $(y=0)$ and bad sleep quality $(y=1)$. Answer 1 to 3 (very good, good, general) was categorized as good sleep quality, answer 4 and 5 (bad and very bad) was categorized as poor sleep quality. The variables including personal monthly income, family monthly income, degree of concerning about their own health, self-reported health status, take medication (i.e. hypnotics), physical activities, smoking, alcohol drinking, having breakfast, eating vegetables, eating fruits, square dancing, daily screen time and using electronic devices before sleep were treated as continuous variables in logistic regression.

\section{Results}




\section{Subjects characteristics and $\mathrm{COR}$}

A total of 11036 subjects were included in the final analysis (363 (3.2\%) subjects were excluded due to missing data or invalid questionnaire). Subjects characteristics and cOR were described in Table 1 grouped by sleep quality. Our study included participants aged from 18 to 90 and with total average of $(46.8 \pm 15.57)$ years. There were $35.4 \%$ males and $59.2 \%$ females. Most men $(89.3 \%)$ and women $(85.8 \%)$ had a good sleep. There were $58.6 \%$ participants with a normal BMI. Most(72.7\%) were married or remarried or cohabiting with boyfriend/girlfriend. Around one third of them were highly educated. Only $13.9 \%$ participants reported a personal monthly income $\geq 4000 \mathrm{RMB}$, and $21.8 \%$ participants reported a family monthly income $\geq 8000 \mathrm{RMB}$. The time of exposure to sunlight was $(164.5 \pm 128.14)$ minutes. Most participants were concerned about their own health, and the majority of them reported a good health status. Only $14.3 \%$ participants had taken medication (e.g. hypnotics such as benzodiazepines or sedatives) to help them to sleep in the last month. Many of them exercised more than 1 time per week. Three quarters were non-smokers, and $68.8 \%$ of all were non-drinkers. More than half had breakfast every day. Almost $60 \%$ participants ate at most $200 \mathrm{~g}$ vegetables each day. Nearly half (46.4\%) of participants ate fruits no more than 2 times per week. Almost one quarter (23.5\%) went square dancing from time to time(occasionally or $\geq 1$ time per week). About one third (32.8\%) used electronic devices more than 3 hours per day, and $44.5 \%$ participants frequently (often, almost everyday) used electronic devices before sleep. There were $17.6 \%$ participants with hypertension, $6.0 \%$ with diabetes, $8.1 \%$ with hyperlipidemia, $6.8 \%$ with heart disease, $2.4 \%$ with respiratory diseases, $1.6 \%$ with stroke, $0.4 \%$ with malignant tumor, $0.6 \%$ with hyperuricemia and $4.3 \%$ with musculoskeletal diseases. 
Table 1

Characteristics of Participants and COR

\begin{tabular}{|c|c|c|c|c|c|}
\hline & Sleep qualii & & Total $(\mathrm{N}=$ & COR & $P$ value \\
\hline & $\begin{array}{l}\text { Good }(N= \\
9628)\end{array}$ & $\begin{array}{l}\text { Poor }(N= \\
1408)\end{array}$ & & & \\
\hline Age (years), (mean \pm SD) & $\begin{array}{l}46.4 \pm \\
15.43\end{array}$ & $\begin{array}{l}49.5 \pm \\
16.27\end{array}$ & $\begin{array}{l}46.8 \pm \\
15.57\end{array}$ & 1.013 & $\hat{0}_{0.001 * \star *}$ \\
\hline Gender, n (\%) & & & & & \\
\hline Male & $\begin{array}{l}3488 \\
(89.3)\end{array}$ & $\begin{array}{l}419 \\
(10.7)\end{array}$ & $\begin{array}{l}3907 \\
(35.4)\end{array}$ & 1.000 & reference \\
\hline Female & $\begin{array}{l}5610 \\
(85.8)\end{array}$ & $\begin{array}{l}928 \\
(14.2)\end{array}$ & $\begin{array}{l}6538 \\
(59.2)\end{array}$ & 1.377 & 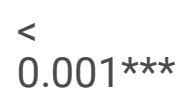 \\
\hline Missing & $530(89.7)$ & $61(10.3)$ & $591(5.4)$ & & \\
\hline BMI, n (\%) & & & & & \\
\hline $\mathrm{BMI}<18.5$ & $634(85.1)$ & $\begin{array}{l}111 \\
(14.9)\end{array}$ & $745(6.8)$ & 1.255 & $0.038 *$ \\
\hline $18.5 \leq \mathrm{BMI} \leq 23.9$ & $\begin{array}{l}5679 \\
(87.8)\end{array}$ & $\begin{array}{l}792 \\
(12.2)\end{array}$ & $\begin{array}{l}6471 \\
(58.6)\end{array}$ & 1.000 & reference \\
\hline $23.9<\mathrm{BMI} \leq 27.9$ & $\begin{array}{l}2630 \\
(86.8)\end{array}$ & $\begin{array}{l}399 \\
(13.2)\end{array}$ & $\begin{array}{l}3029 \\
(27.4)\end{array}$ & 1.088 & 0.201 \\
\hline $\mathrm{BMI}>27.9$ & $536(85.5)$ & 91 (14.5) & $627(5.7)$ & 1.217 & 0.100 \\
\hline Missing & $149(90.9)$ & $15(9.2)$ & $164(1.5)$ & & \\
\hline Marital status, n (\%) & & & & & \\
\hline $\begin{array}{l}\text { Unmarried and without cohabiting } \\
\text { with boyfriend / girlfriend }\end{array}$ & 753 (86.4) & $\begin{array}{l}118 \\
(13.6)\end{array}$ & $871(7.9)$ & 1.110 & 0.320 \\
\hline $\begin{array}{l}\text { Married or remarried or cohabiting } \\
\text { with boyfriend / girlfriend }\end{array}$ & $\begin{array}{l}7032 \\
(87.6)\end{array}$ & $\begin{array}{l}993 \\
(12.4)\end{array}$ & $\begin{array}{l}8025 \\
(72.7)\end{array}$ & 1.000 & reference \\
\hline Divorced or widowed & $310(77.9)$ & $88(22.1)$ & $398(3.6)$ & 2.010 & $<.001 * \star \star$ \\
\hline Missing & $\begin{array}{l}1533 \\
(88.0)\end{array}$ & $\begin{array}{l}209 \\
(12.0)\end{array}$ & $\begin{array}{l}1742 \\
(15.8)\end{array}$ & & \\
\hline
\end{tabular}

n, number;

cOR was analyzed with one-way logistic regression;

$*:<0.05, * *:<0.01, * \star *:<0.001$. 


\begin{tabular}{|c|c|c|c|c|c|}
\hline & \multicolumn{2}{|c|}{ Sleep quality } & \multirow{2}{*}{$\begin{array}{l}\text { Total }(\mathrm{N}= \\
11036)\end{array}$} & \multirow[t]{2}{*}{ cOR } & \multirow[t]{2}{*}{$P$ value } \\
\hline & $\begin{array}{l}\text { Good }(N= \\
9628)\end{array}$ & $\begin{array}{l}\text { Poor }(N= \\
1408)\end{array}$ & & & \\
\hline Primary school and below & $\begin{array}{l}1809 \\
(82.4)\end{array}$ & $\begin{array}{l}387 \\
(17.6)\end{array}$ & $\begin{array}{l}2196 \\
(19.9)\end{array}$ & 1.560 & $\begin{array}{l}<.001^{\star \star \star *} \\
0\end{array}$ \\
\hline Middle/ high school & $\begin{array}{l}4524 \\
(88.7)\end{array}$ & $\begin{array}{l}575 \\
(11.3)\end{array}$ & $\begin{array}{l}5099 \\
(46.2)\end{array}$ & 0.927 & 0.259 \\
\hline College and above & $\begin{array}{l}3186 \\
(87.9)\end{array}$ & $\begin{array}{l}437 \\
(12.1)\end{array}$ & $\begin{array}{l}3623 \\
(32.8)\end{array}$ & 1.000 & reference \\
\hline Missing & $109(92.4)$ & $9(7.6)$ & $118(1.1)$ & & \\
\hline Personal monthly income, $\mathrm{n}(\%)$ & & & & 0.896 & $\begin{array}{l}<.001 * \star \star \\
0.0\end{array}$ \\
\hline $0 ¥$ & $\begin{array}{l}2073 \\
(83.7)\end{array}$ & $\begin{array}{l}404 \\
(16.3)\end{array}$ & $\begin{array}{l}2477 \\
(22.4)\end{array}$ & & \\
\hline $1-999 ¥$ & $\begin{array}{l}1001 \\
(83.1)\end{array}$ & $\begin{array}{l}203 \\
(16.9)\end{array}$ & $\begin{array}{l}1204 \\
(10.9)\end{array}$ & & \\
\hline $1000-1999 ¥$ & $\begin{array}{l}1808 \\
(87.9)\end{array}$ & $\begin{array}{l}248 \\
(12.1)\end{array}$ & $\begin{array}{l}2056 \\
(18.6)\end{array}$ & & \\
\hline $2000-2999 ¥$ & $\begin{array}{l}1985 \\
(90.6)\end{array}$ & $205(9.4)$ & $\begin{array}{l}2190 \\
(19.8)\end{array}$ & & \\
\hline $3000-3999 ¥$ & $\begin{array}{l}1272 \\
(88.7)\end{array}$ & $\begin{array}{l}162 \\
(11.3)\end{array}$ & $\begin{array}{l}1434 \\
(13.0)\end{array}$ & & \\
\hline $4000-4999 ¥$ & $793(88.6)$ & $\begin{array}{l}102 \\
(11.4)\end{array}$ & $895(8.1)$ & & \\
\hline $5000-5999 ¥$ & $311(89.1)$ & $38(10.9)$ & $349(3.2)$ & & \\
\hline $6000-6999 ¥$ & $130(90.3)$ & $14(9.7)$ & $144(1.3)$ & & \\
\hline$\geq 7000 ¥$ & $132(91.7)$ & $12(8.3)$ & $144(1.3)$ & & \\
\hline Missing & $123(86)$ & $20(14)$ & $143(1.3)$ & & \\
\hline Family monthly income, $\mathrm{n}(\%)$ & & & & 0.896 & $\begin{array}{l}<.001^{\star \star *} \\
\end{array}$ \\
\hline $0 ¥$ & $700(83.0)$ & $\begin{array}{l}143 \\
(17.0)\end{array}$ & $843(7.6)$ & & \\
\hline
\end{tabular}

n, number;

cOR was analyzed with one-way logistic regression;

$*:<0.05, * *:<0.01, * \star *:<0.001$. 


\begin{tabular}{|c|c|c|c|c|c|}
\hline & Sleep quali & & Total $(\mathrm{N}=$ & COR & $P$ value \\
\hline & $\begin{array}{l}\text { Good }(N= \\
9628)\end{array}$ & $\begin{array}{l}\text { Poor }(N= \\
1408)\end{array}$ & & & \\
\hline $1-1999 ¥$ & $\begin{array}{l}1123 \\
(83.3)\end{array}$ & $\begin{array}{l}225 \\
(16.7)\end{array}$ & $\begin{array}{l}1348 \\
(12.2)\end{array}$ & & \\
\hline $2000-3999 ¥$ & $\begin{array}{l}2006 \\
(85.5)\end{array}$ & $\begin{array}{l}339 \\
(14.5)\end{array}$ & $\begin{array}{l}2345 \\
(21.2)\end{array}$ & & \\
\hline $4000-5999 ¥$ & $\begin{array}{l}1976 \\
(89.1)\end{array}$ & $\begin{array}{l}242 \\
(10.9)\end{array}$ & $\begin{array}{l}2218 \\
(20.1)\end{array}$ & & \\
\hline $6000-7999 ¥$ & $\begin{array}{l}1297 \\
(88.7)\end{array}$ & $\begin{array}{l}165 \\
(11.3)\end{array}$ & $\begin{array}{l}1462 \\
(13.2)\end{array}$ & & \\
\hline 8000-9999¥ & $859(88.8)$ & $\begin{array}{l}108 \\
(11.2)\end{array}$ & $967(8.8)$ & & \\
\hline $10000-11999 ¥$ & $634(90.1)$ & $70(9.9)$ & $704(6.4)$ & & \\
\hline $12000-13999 ¥$ & $308(90.9)$ & $31(9.1)$ & $339(3.1)$ & & \\
\hline$\geq 14000 ¥$ & $358(91.1)$ & $35(8.9)$ & $393(3.6)$ & & \\
\hline Missing & $367(88)$ & $50(12)$ & $417(3.8)$ & & \\
\hline $\begin{array}{l}\text { Time of exposure to sunlight } \\
\text { (minutes), (mean } \pm S D \text { ) }\end{array}$ & $\begin{array}{l}165.1 \pm \\
128.05\end{array}$ & $\begin{array}{l}160.3 \pm \\
128.73\end{array}$ & $\begin{array}{l}164.5 \pm \\
128.14\end{array}$ & 1.000 & 0.212 \\
\hline Missing, n (\%) & $748(84.2)$ & $\begin{array}{l}140 \\
(15.8)\end{array}$ & $888(8.0)$ & & \\
\hline $\begin{array}{l}\text { Degree of concerning about their own } \\
\text { health, } n(\%)\end{array}$ & & & & 0.915 & $0.007 *$ \\
\hline Very unconcerned & $76(80.0)$ & $19(20.0)$ & $95(0.9)$ & & \\
\hline Unconcerned & $254(80.9)$ & $60(19.1)$ & $314(2.9)$ & & \\
\hline General & $\begin{array}{l}2373 \\
(87.2)\end{array}$ & $\begin{array}{l}347 \\
(12.8)\end{array}$ & $\begin{array}{l}2720 \\
(24.6)\end{array}$ & & \\
\hline Concerned & $\begin{array}{l}3874 \\
(87.4)\end{array}$ & $\begin{array}{l}561 \\
(12.6)\end{array}$ & $\begin{array}{l}4435 \\
(40.2)\end{array}$ & & \\
\hline Very concerned & $\begin{array}{l}2952 \\
(87.9)\end{array}$ & $\begin{array}{l}407 \\
(12.1)\end{array}$ & $\begin{array}{l}3359 \\
(30.4)\end{array}$ & & \\
\hline Missing & $99(87.6)$ & $14(12.4)$ & $113(1.0)$ & & \\
\hline n, number; & & & & & \\
\hline cOR was analyzed with one-way logist & regression; & & & & \\
\hline$\star:<0.05, * *:<0.01, * \star *:<0.001$. & & & & & \\
\hline
\end{tabular}




\begin{tabular}{|c|c|c|c|c|c|}
\hline & Sleep quali & & Total $(\mathrm{N}=$ & COR & $P$ value \\
\hline & $\begin{array}{l}\text { Good }(N= \\
9628)\end{array}$ & $\begin{array}{l}\text { Poor }(N= \\
1408)\end{array}$ & & & \\
\hline Self-reported health status, n (\%) & & & & 0.459 & $<.001 * \star *$ \\
\hline Very bad & $66(53.2)$ & $58(46.8)$ & $124(1.1)$ & & \\
\hline Bad & $447(64.4)$ & $\begin{array}{l}247 \\
(35.6)\end{array}$ & $694(6.3)$ & & \\
\hline General & $\begin{array}{l}3448 \\
(84.3)\end{array}$ & $\begin{array}{l}641 \\
(15.7)\end{array}$ & $\begin{array}{l}4089 \\
(37.0)\end{array}$ & & \\
\hline Good & $\begin{array}{l}4196 \\
(91.7)\end{array}$ & $379(8.3)$ & $\begin{array}{l}4575 \\
(41.5)\end{array}$ & & \\
\hline Very good & $\begin{array}{l}1366 \\
(94.6)\end{array}$ & $78(5.4)$ & $\begin{array}{l}1444 \\
(13.1)\end{array}$ & & \\
\hline Missing & $105(95.5)$ & $5(4.6)$ & $110(1.0)$ & & \\
\hline Take medication (i.e. hypnotics), n (\%) & & & & 1.849 & 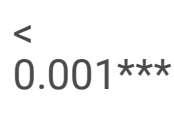 \\
\hline Never & $\begin{array}{l}8316 \\
(89.7)\end{array}$ & $\begin{array}{l}956 \\
(10.3)\end{array}$ & $\begin{array}{l}9272 \\
(84.0)\end{array}$ & & \\
\hline Rarely & $715(78.8)$ & $\begin{array}{l}192 \\
(21.2)\end{array}$ & 907 (8.2) & & \\
\hline Sometimes & $274(70.4)$ & $\begin{array}{l}115 \\
(29.6)\end{array}$ & $389(3.5)$ & & \\
\hline Often & $121(56.3)$ & $94(43.7)$ & $215(2.0)$ & & \\
\hline Always & $36(59.0)$ & $25(41.0)$ & $61(0.6)$ & & \\
\hline Missing & $166(86.5)$ & $26(13.5)$ & $192(1.7)$ & & \\
\hline Physical activities, n (\%) & & & & 1.009 & 0.761 \\
\hline 0 time per week & $\begin{array}{l}2075 \\
(86.0)\end{array}$ & $\begin{array}{l}338 \\
(14.0)\end{array}$ & $\begin{array}{l}2413 \\
(21.9)\end{array}$ & & \\
\hline 1-2 times per week & $\begin{array}{l}3807 \\
(88.6)\end{array}$ & $\begin{array}{l}491 \\
(11.4)\end{array}$ & $\begin{array}{l}4298 \\
(38.9)\end{array}$ & & \\
\hline
\end{tabular}

n, number;

cOR was analyzed with one-way logistic regression;

$\star:<0.05, * *:<0.01, * \star *:<0.001$. 


\begin{tabular}{|c|c|c|c|c|c|}
\hline & Sleep quali & & Total $(\mathrm{N}=$ & cOR & $P$ value \\
\hline & $\begin{array}{l}\text { Good }(N= \\
9628)\end{array}$ & $\begin{array}{l}\text { Poor }(N= \\
1408)\end{array}$ & & & \\
\hline 3-4 times per week & $\begin{array}{l}2065 \\
(87.1)\end{array}$ & $\begin{array}{l}305 \\
(12.9)\end{array}$ & $\begin{array}{l}2370 \\
(21.5)\end{array}$ & & \\
\hline$\geq 5$ times per week & $\begin{array}{l}1562 \\
(86.2)\end{array}$ & $\begin{array}{l}249 \\
(13.8)\end{array}$ & $\begin{array}{l}1811 \\
(16.4)\end{array}$ & & \\
\hline Missing & $119(82.6)$ & $25(17.4)$ & $144(1.3)$ & & \\
\hline Smoking, n (\%) & & & & 1.077 & $0.012^{\star}$ \\
\hline Non-smokers & $\begin{array}{l}7260 \\
(87.3)\end{array}$ & $\begin{array}{l}1056 \\
(12.7)\end{array}$ & $\begin{array}{l}8316 \\
(75.3)\end{array}$ & & \\
\hline Occasional smokers & $972(90.9)$ & $97(9.1)$ & $1069(9.7)$ & & \\
\hline $1-10$ cigarettes per day & $685(84.4)$ & $\begin{array}{l}127 \\
(15.6)\end{array}$ & $812(7.4)$ & & \\
\hline $11-20$ cigarettes per day & $375(84.8)$ & $67(15.2)$ & $442(4.0)$ & & \\
\hline > 20 cigarettes per day & $164(82.0)$ & $36(18.0)$ & $200(1.8)$ & & \\
\hline Missing & $172(87.3)$ & $25(12.7)$ & $197(1.8)$ & & \\
\hline Alcohol drinking, n (\%) & & & & 1.045 & 0.160 \\
\hline Non-drinkers & $\begin{array}{l}6659 \\
(87.7)\end{array}$ & $\begin{array}{l}937 \\
(12.3)\end{array}$ & $\begin{array}{l}7596 \\
(68.8)\end{array}$ & & \\
\hline Less than 1 time per month & $\begin{array}{l}1314 \\
(84.8)\end{array}$ & $\begin{array}{l}236 \\
(15.2)\end{array}$ & $\begin{array}{l}1550 \\
(14.0)\end{array}$ & & \\
\hline $1-3$ times per month & $\begin{array}{l}1260 \\
(89.1)\end{array}$ & $\begin{array}{l}154 \\
(10.9)\end{array}$ & $\begin{array}{l}1414 \\
(12.8)\end{array}$ & & \\
\hline $1-3$ times per week & $266(83.9)$ & $51(16.1)$ & $317(2.9)$ & & \\
\hline$\geq 4$ times per week & $88(81.5)$ & $20(18.5)$ & $108(1.0)$ & & \\
\hline Missing & $41(80.4)$ & $10(19.6)$ & $51(0.5)$ & & \\
\hline Having breakfast, n (\%) & & & & 0.953 & $0.022^{*}$ \\
\hline 0 time per week & $770(85.4)$ & $\begin{array}{l}132 \\
(14.6)\end{array}$ & $902(8.2)$ & & \\
\hline
\end{tabular}

n, number;

cOR was analyzed with one-way logistic regression;

$\star:<0.05, * *:<0.01, * \star *:<0.001$. 


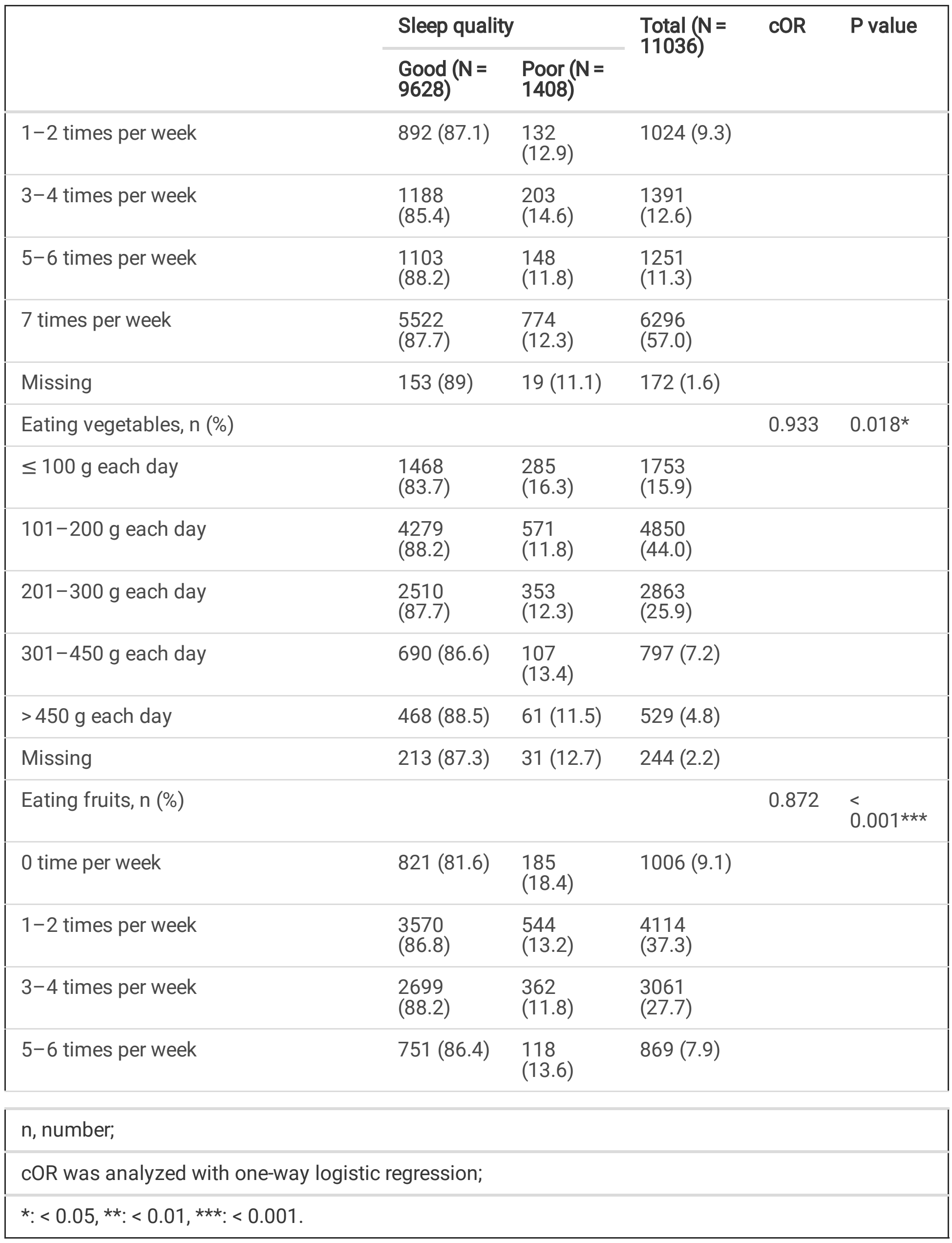




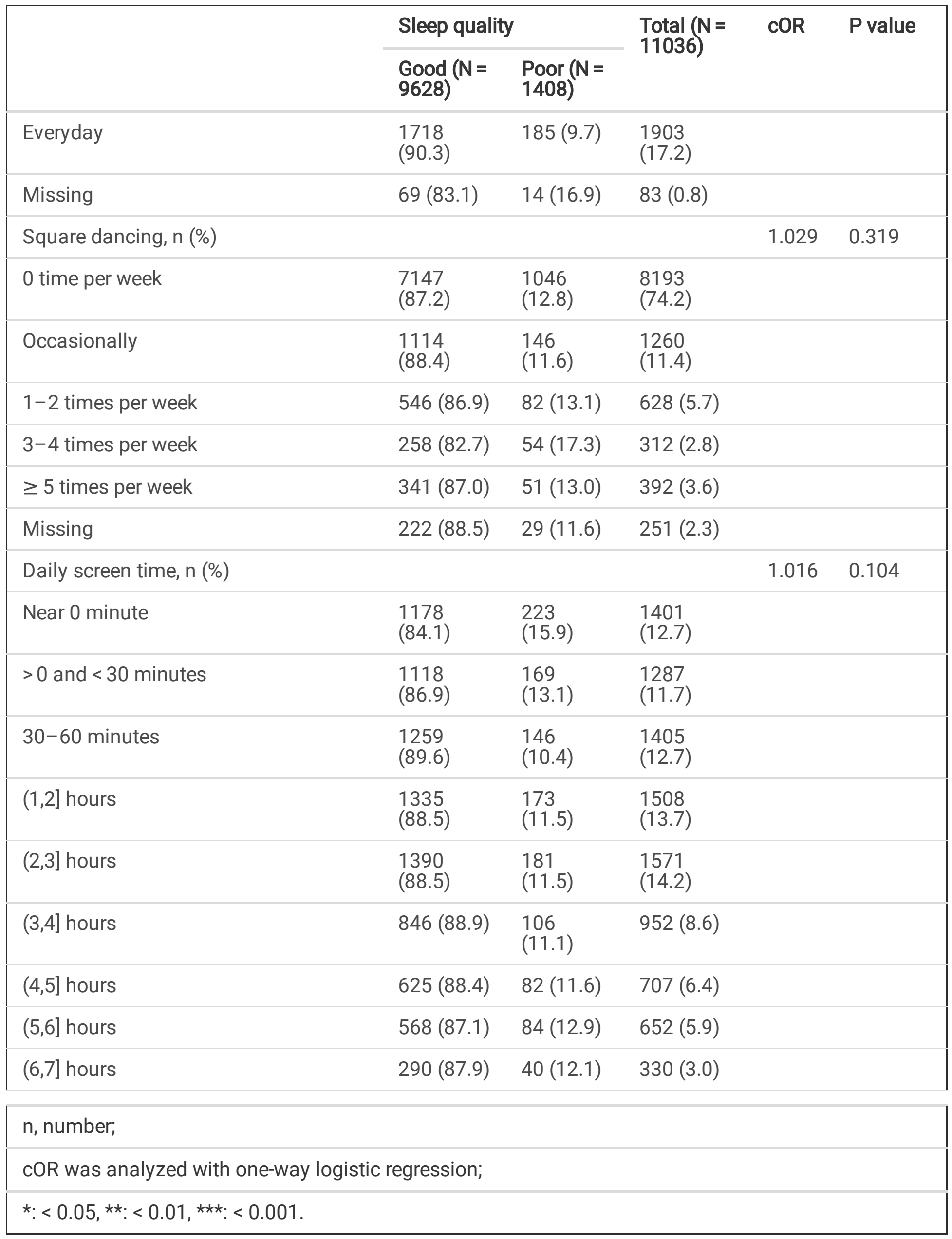




\begin{tabular}{|c|c|c|c|c|c|}
\hline & \multicolumn{2}{|c|}{ Sleep quality } & \multirow{2}{*}{$\begin{array}{l}\text { Total }(\mathrm{N}= \\
11036)\end{array}$} & \multirow[t]{2}{*}{ COR } & \multirow[t]{2}{*}{$P$ value } \\
\hline & $\begin{array}{l}\text { Good }(N= \\
9628)\end{array}$ & $\begin{array}{l}\text { Poor }(N= \\
1408)\end{array}$ & & & \\
\hline$(7,8]$ hours & 208 (85.3) & $36(14.8)$ & $244(2.2)$ & & \\
\hline$(8,9]$ hours & 227 (82.0) & $50(18.0)$ & $277(2.5)$ & & \\
\hline$\geq 9$ hours & 367 (81.0) & $86(19.0)$ & $453(4.1)$ & & \\
\hline Missing & 217 (87.2) & $32(12.9)$ & $249(2.3)$ & & \\
\hline $\begin{array}{l}\text { Using electronic devices before sleep, } \\
\text { n (\%) }\end{array}$ & & & & 1.045 & $0.038^{*}$ \\
\hline Never & $\begin{array}{l}1438 \\
(84.6)\end{array}$ & $\begin{array}{l}261 \\
(15.4)\end{array}$ & $\begin{array}{l}1699 \\
(15.4)\end{array}$ & & \\
\hline Rarely & $\begin{array}{l}1667 \\
(90.2)\end{array}$ & $182(9.8)$ & $\begin{array}{l}1849 \\
(16.7)\end{array}$ & & \\
\hline Sometimes & $\begin{array}{l}2147 \\
(89.3)\end{array}$ & $\begin{array}{l}257 \\
(10.7)\end{array}$ & $\begin{array}{l}2404 \\
(21.8)\end{array}$ & & \\
\hline Often & $\begin{array}{l}2331 \\
(87.8)\end{array}$ & $\begin{array}{l}325 \\
(12.2)\end{array}$ & $\begin{array}{l}2656 \\
(24.1)\end{array}$ & & \\
\hline Almost everyday & $\begin{array}{l}1889 \\
(84.0)\end{array}$ & $\begin{array}{l}360 \\
(16.0)\end{array}$ & $\begin{array}{l}2249 \\
(20.4)\end{array}$ & & \\
\hline Missing & 156 (87.2) & $23(12.9)$ & $179(1.6)$ & & \\
\hline \multicolumn{6}{|l|}{ Hypertension, n (\%) } \\
\hline Yes & $\begin{array}{l}1623 \\
(83.4)\end{array}$ & $\begin{array}{l}324 \\
(16.6)\end{array}$ & $\begin{array}{l}1947 \\
(17.6)\end{array}$ & 1.475 & $\begin{array}{l}<.001 * \star \star \\
\end{array}$ \\
\hline No & $\begin{array}{l}7804 \\
(88.1)\end{array}$ & $\begin{array}{l}1056 \\
(11.9)\end{array}$ & $\begin{array}{l}8860 \\
(80.3)\end{array}$ & 1.000 & reference \\
\hline Missing & 201 (87.8) & $28(12.2)$ & $229(2.1)$ & & \\
\hline \multicolumn{6}{|l|}{ Diabetes, n (\%) } \\
\hline Yes & $521(78.5)$ & $\begin{array}{l}143 \\
(21.5)\end{array}$ & $664(6.0)$ & 1.974 & $\begin{array}{l}<.001 * \star \star \\
\end{array}$ \\
\hline No & $\begin{array}{l}8910 \\
(87.8)\end{array}$ & $\begin{array}{l}1239 \\
(12.2)\end{array}$ & $\begin{array}{l}10149 \\
(92.0)\end{array}$ & 1.000 & reference \\
\hline
\end{tabular}

n, number;

cOR was analyzed with one-way logistic regression;

$*:<0.05, * *:<0.01, * \star *:<0.001$. 


\begin{tabular}{|c|c|c|c|c|c|}
\hline & \multicolumn{2}{|c|}{ Sleep quality } & \multirow{2}{*}{$\begin{array}{l}\text { Total }(\mathrm{N}= \\
11036)\end{array}$} & \multirow[t]{2}{*}{ COR } & \multirow[t]{2}{*}{$P$ value } \\
\hline & $\begin{array}{l}\text { Good }(\mathrm{N}= \\
9628)\end{array}$ & $\begin{array}{l}\text { Poor }(N= \\
1408)\end{array}$ & & & \\
\hline Missing & $197(88.3)$ & $26(11.7)$ & $223(2.0)$ & & \\
\hline \multicolumn{6}{|c|}{ Hyperlipidemia, n (\%) } \\
\hline Yes & 725 (81.4) & $\begin{array}{l}166 \\
(18.6)\end{array}$ & $891(8.1)$ & 1.639 & $\begin{array}{l}<.001 * \star \star \\
0.01\end{array}$ \\
\hline No & $\begin{array}{l}8707 \\
(87.8)\end{array}$ & $\begin{array}{l}1216 \\
(12.2)\end{array}$ & $\begin{array}{l}9923 \\
(89.9)\end{array}$ & 1.000 & reference \\
\hline Missing & $196(88.3)$ & $26(11.7)$ & $222(2.0)$ & & \\
\hline \multicolumn{6}{|c|}{ Heart disease, n (\%) } \\
\hline Yes & $556(74.7)$ & $\begin{array}{l}188 \\
(25.3)\end{array}$ & $744(6.8)$ & 2.513 & $\hat{0}_{0.001 * \star \star}$ \\
\hline No & $\begin{array}{l}8875 \\
(88.1)\end{array}$ & $\begin{array}{l}1194 \\
(11.9)\end{array}$ & $\begin{array}{l}10069 \\
(91.2)\end{array}$ & 1.000 & reference \\
\hline Missing & $197(88.3)$ & $26(11.7)$ & $223(2.0)$ & & \\
\hline \multicolumn{6}{|c|}{ Respiratory diseases, n (\%) } \\
\hline Yes & $196(75.1)$ & 65 (24.9) & $261(2.4)$ & 2.326 & $\stackrel{<}{<.001^{\star \star \star *}}$ \\
\hline No & $\begin{array}{l}9235 \\
(87.5)\end{array}$ & $\begin{array}{l}1317 \\
(12.5)\end{array}$ & $\begin{array}{l}10552 \\
(95.6)\end{array}$ & 1.000 & reference \\
\hline Missing & 197 (88.3) & $26(11.7)$ & $223(2.0)$ & & \\
\hline \multicolumn{6}{|c|}{ Stroke, n (\%) } \\
\hline Yes & $129(75.9)$ & $41(24.1)$ & $170(1.6)$ & 2.205 & $\begin{array}{l}<.001 * \star \star \\
0.0\end{array}$ \\
\hline No & $\begin{array}{l}9302 \\
(87.4)\end{array}$ & $\begin{array}{l}1341 \\
(12.6)\end{array}$ & $\begin{array}{l}10643 \\
(96.4)\end{array}$ & 1.000 & reference \\
\hline Missing & $197(88.3)$ & $26(11.7)$ & $223(2.0)$ & & \\
\hline \multicolumn{6}{|c|}{ Malignant tumor, n (\%) } \\
\hline Yes & $34(73.9)$ & $12(26.1)$ & $46(0.4)$ & 2.421 & $0.009 *$ \\
\hline
\end{tabular}

n, number;

cOR was analyzed with one-way logistic regression;

$\star:<0.05, * *:<0.01, * \star *:<0.001$. 


\begin{tabular}{|c|c|c|c|c|c|}
\hline & \multicolumn{2}{|c|}{ Sleep quality } & \multirow{2}{*}{$\begin{array}{l}\text { Total }(\mathrm{N}= \\
11036)\end{array}$} & \multirow[t]{2}{*}{ COR } & \multirow[t]{2}{*}{$P$ value } \\
\hline & $\begin{array}{l}\text { Good (N= } \\
9628)\end{array}$ & $\begin{array}{l}\text { Poor }(N= \\
1408)\end{array}$ & & & \\
\hline No & $\begin{array}{l}9397 \\
(87.3)\end{array}$ & $\begin{array}{l}1370 \\
(12.7)\end{array}$ & $\begin{array}{l}10767 \\
(97.6)\end{array}$ & 1.000 & reference \\
\hline Missing & $197(88.3)$ & $26(11.7)$ & $223(2.0)$ & & \\
\hline \multicolumn{6}{|l|}{ Hyperuricemia, n (\%) } \\
\hline Yes & $54(78.3)$ & $15(21.7)$ & $69(0.6)$ & 1.905 & $0.028^{*}$ \\
\hline No & $\begin{array}{l}9377 \\
(87.3)\end{array}$ & $\begin{array}{l}1367 \\
(12.7)\end{array}$ & $\begin{array}{l}10744 \\
(97.4)\end{array}$ & 1.000 & reference \\
\hline Missing & $197(88.3)$ & $26(11.7)$ & $223(2.0)$ & & \\
\hline \multicolumn{6}{|l|}{ Musculoskeletal diseases, n (\%) } \\
\hline Yes & $361(75.7)$ & $\begin{array}{l}116 \\
(24.3)\end{array}$ & $477(4.3)$ & 2.302 & $\begin{array}{l}<.001 * \star \star \\
\end{array}$ \\
\hline No & $\begin{array}{l}9070 \\
(87.8)\end{array}$ & $\begin{array}{l}1266 \\
(12.2)\end{array}$ & $\begin{array}{l}10336 \\
(93.7)\end{array}$ & 1.000 & reference \\
\hline Missing & $197(88.3)$ & $26(11.7)$ & $223(2.0)$ & & \\
\hline \multicolumn{6}{|l|}{ n, number; } \\
\hline \multicolumn{6}{|c|}{ cOR was analyzed with one-way logistic regression; } \\
\hline$*:<0.05, * *:<0.01, * \star *:<0.001$ & & & & & \\
\hline
\end{tabular}

The cOR were shown as below table. Poorer sleep quality was associated with older age $(P<0.001)$, female $(P<0.001), B M I<18.5(P=0.038)$, divorced or widowed $(P<0.001)$, primary school or below educational level $(P<0.001)$, take hypnotics $(P<0.001)$, smoking $(P=0.012)$, using electronic devices before sleep frequently $(P=0.038)$, hypertension $(P<0.001)$, diabetes $(P<0.001)$, hyperlipidemia $(P<$ $0.001)$, heart disease $(P<0.001)$, respiratory diseases $(P<0.001)$, stroke $(P<0.001)$, malignant tumor $(P=$ 0.009), hyperuricemia $(P=0.028)$, musculoskeletal diseases $(P<0.001)$, while better sleep was associated with high personal monthly income $(P<0.001)$, high family monthly income $(P<0.001)$, concerning about their own health $(P=0.007)$, good self-reported health status $(P<0.001)$, having breakfast regularly $(P=0.022)$, eating more vegetables $(P=0.018)$, eating more fruits $(P<0.001)$.

Table 1. Characteristics of Participants and cOR (refer to the end of the manuscript).

\section{Sleep status}

In this study, $50.7 \%$ participants had a good or very good sleep, $36.5 \%$ participants' self-reported sleep quality was general, and $12.8 \%$ participants had a bad or very bad sleep. In the last month, $8.4 \%$ 
participants had difficulty in initiating sleep $\geq 3$ times per week, $7.6 \%$ participants had difficulty in maintaining sleep $\geq 3$ times per week, $8.8 \%$ participants suffered from awakening earlier $\geq 3$ times per week, $10.3 \%$ participants had the problem of feeling sleepy during the day $\geq 3$ times per week. There were $83.5 \%$ participants having one of the above sleep problems (difficulty initiating sleep, difficulty maintaining sleep, awakening early) $\leq 3$ months, and $16.5 \%$ participants having one of the above sleep problems $>3$ months. The detailed information of sleep status was described in Table 2 . 
Table 2

Sleep Status of Participants

\begin{tabular}{|c|c|c|}
\hline & Frequency & Percentage (\%) \\
\hline \multicolumn{3}{|c|}{ Self-reported sleep quality } \\
\hline Very good & 1619 & 14.7 \\
\hline Good & 3977 & 36.0 \\
\hline General & 4032 & 36.5 \\
\hline Bad & 1179 & 10.7 \\
\hline Very bad & 229 & 2.1 \\
\hline \multicolumn{3}{|l|}{ Difficulty initiating sleep } \\
\hline Never & 4698 & 42.6 \\
\hline $1-3$ times per month & 3622 & 32.8 \\
\hline 1-2 times per week & 1698 & 15.4 \\
\hline 3-4 times per week & 574 & 5.2 \\
\hline$\geq 5$ times per week & 353 & 3.2 \\
\hline Missing & 91 & 0.8 \\
\hline \multicolumn{3}{|c|}{ Difficulty maintaining sleep } \\
\hline Never & 5098 & 46.2 \\
\hline $1-3$ times per month & 3349 & 30.4 \\
\hline 1-2 times per week & 1557 & 14.1 \\
\hline 3-4 times per week & 546 & 4.9 \\
\hline$\geq 5$ times per week & 271 & 2.5 \\
\hline Missing & 215 & 1.9 \\
\hline \multicolumn{3}{|l|}{ Awakening earlier } \\
\hline Never & 4923 & 44.6 \\
\hline $1-3$ times per month & 3342 & 30.3 \\
\hline $1-2$ times per week & 1629 & 14.7 \\
\hline 3-4 times per week & 560 & 5.1 \\
\hline$\geq 5$ times per week & 394 & 3.6 \\
\hline
\end{tabular}




\begin{tabular}{|lll|}
\hline & Frequency & Percentage (\%) \\
\hline Missing & 188 & 1.7 \\
\hline Feeling sleepy during the day & & \\
\hline Never & 4178 & 37.9 \\
\hline $1-3$ times per month & 3401 & 30.8 \\
\hline $1-2$ times per week & 2073 & 18.8 \\
\hline $3-4$ times per week & 680 & 6.1 \\
\hline$\geq 5$ times per week & 431 & 3.9 \\
\hline Missing & 273 & 2.5 \\
\hline How long have you had one of above sleep problems & & \\
\hline Never & 4889 & 44.3 \\
\hline$>0$ - 2 weeks & 2498 & 22.6 \\
\hline 2 weeks-1 month & 1017 & 9.2 \\
\hline$>1$ month-3 months & 592 & 5.4 \\
\hline$>3$ months-6 months & 339 & 3.1 \\
\hline$>6$ months-12 months & 392 & 3.6 \\
\hline$>12$ months & 1050 & 9.5 \\
\hline Missing & 259 & 2.3 \\
\hline
\end{tabular}

Table 2. Sleep Status of Participants (refer to the end of the manuscript).

\section{The associated factors of sleep quality}

The associated factors obtained by stepwise regression analysis were shown in the Table 3. In our study, female participants slept poorer than male participants $(a O R=1.792, p<0.001)$. Subjects having a life partner slept better than those without life partner, the aOR of unmarried or without cohabiting with boyfriend/girlfriend was $1.319(95 \% \mathrm{Cl}=1.022-1.704)$, and the aOR of divorced or widowed was 1.436 $(95 \% \mathrm{Cl}=1.052-1.960)$. The highly educated (college and above) participants slept worse than those less educated (middle/ high school) $(\mathrm{aOR}=0.748, \mathrm{p}=0.003)$. Participants slept better with increased family monthly income $(\mathrm{aOR}=0.939, \mathrm{p}=0.004)$. Subjects with chronic noncommunicable diseases such as heart disease $(\mathrm{aOR}=1.478, \mathrm{p}=0.002)$ and musculoskeletal diseases $(\mathrm{aOR}=1.460, \mathrm{p}=0.018)$ had a poorer sleep. Participants slept poorer if they were more concerned about their own health $(\mathrm{aOR}=1.113$, $p=0.016)$, but sleep better while they reported a better health status $(a O R=0.490, p<0.001)$. With the increase in frequency of alcohol drinking $(a O R=1.201, p<0.001)$, subjects slept more poorer. Participants 
eating breakfast regularly $(\mathrm{aOR}=0.935, \mathrm{p}=0.016)$ had a better sleep. Subjects who took hypnotics more frequently $(\mathrm{aOR}=1.643, \mathrm{p}<0.001)$ had a poorer sleep. People had a poorer sleep while they had a longer daily screen time $(a O R=1.055, p<0.001)$. 
Table 3

Associated Factors of Sleep Quality

Associated factors

Gender

Male (reference)

Female

Marital status

Married or remarried or cohabiting with boyfriend / girlfriend (reference)

Unmarried and without cohabiting with boyfriend / girlfriend

Divorced or widowed

Educational level

College and above (reference)

Middle/ high school

Primary school and below

Family monthly income

Heart disease

Musculoskeletal diseases

Degree of concerning about their own health

Self-reported health status

Alcohol drinking

Having breakfast

Take medication (i.e. hypnotics)

$\star:<0.05, * *:<0.01, * \star *:<0.001$.
Coefficient P value aOR $95 \% \mathrm{Cl}$

$\begin{array}{llll}- & - & - & - \\ 0.583 & < & 1.792 & (1.497, \\ & 0.001 \star \star \star & & 2.144)^{\prime}\end{array}$

$\begin{array}{llll}- & - & - & - \\ 0.277 & 0.034^{*} & 1.319 & \begin{array}{l}(1.022 \\ 1.704)\end{array} \\ 0.362 & 0.023^{*} & 1.436 & \begin{array}{l}(1.052 \\ 1.960)\end{array}\end{array}$

$\begin{array}{lllllll}- & - & - & - & - & -\end{array}$

$0.003^{\star *}$

0.748

$(0.616$ $0.908)$

0.067

0.580

1.069

$(0.843$ 1.357)

$\begin{array}{llll}-0.063 & 0.004^{* *} & 0.939 & (0.900\end{array}$

$0.980)$

$(1.155$, 1.891)

$\begin{array}{llll}0.391 & 0.002 * * & 1.478 & (1.155 \\ & & & 1.891)\end{array}$

0.378

$0.018^{*} \quad 1.460$

(1.067, 1.997)

$\begin{array}{llll}0.107 & 0.016 * & 1.113 & (1.020 \\ & & & 1.214)\end{array}$

$-0.714$

0.490

(0.445, $\left.0.001^{\star * *} \quad 0.490 \quad 0.539\right)$

0.183

$0.001 * \star \star$

1.201

(1.097, 1.315)

$-0.067$

0.016 *

0.935

(0.886, $0.988)$

0.496

$\begin{array}{lll}< & 1.643 \quad(1.498, \\ 0.001 \star \star \star & & 1.801)\end{array}$




\begin{tabular}{|c|c|c|c|c|}
\hline Associated factors & Coefficient & $P$ value & $\mathrm{aOR}$ & $95 \% \mathrm{Cl}$ \\
\hline Daily screen time & 0.053 & $\begin{array}{l}<.001^{\star \star * *} \\
\end{array}$ & 1.055 & $\begin{array}{l}(1.027, \\
1.083)^{\prime}\end{array}$ \\
\hline
\end{tabular}

\section{Discussion}

In our study, self-reported sleep quality was generally good in Shaanxi, China.

From this study, we found that sleep quality was associated with gender, marital status, educational level, family monthly income, heart disease, musculoskeletal diseases, degree of concerning about their own health, self-reported health status, alcohol drinking, having breakfast, take medication (i.e. hypnotics) and daily screen time.

In our study, female participants sleep poorly than male participants. A descriptive, cross-sectional study conducted in Korea reported a consistent conclusion that female was a significant risk factor of poor sleep quality[16]. This may be because women tend to experience a greater prevalence of anxiety disorders [17]. Participants have a life partner sleep better than those without life partner. The reason may be that life partner can provide emotional support to help them sleep well. A 4-year follow-up study in a Korean cohort reported that there are bidirectional relationships between marital status and sleep problems, on the one hand, poor marital quality is a risk factor for sleep disturbance for older adults, on the other hand, sleep disturbance may have a negative impact on marital quality for all age groups[18]. A study suggested that older population with widowed, divorced or separated marital status were more likely to have sleep complaint[19]. This result was consistent with our study. Subjects with medium education level sleep better than highly educated and less educated subjects. The possible reason is that highly educated population may have higher work stress (such as more intense competition in daily work), while less educated people may face higher survival stress (such as lower salary). With the increase in family monthly income, participants sleep better. But the personal monthly income wasn't a significant factor, that's probably because family members always live together in China. A longitudinal community study named Ibadan Study of Ageing found that decreasing economic status had an association with increasing incidence of insomnia[20]. Participants with chronic noncommunicable diseases such as heart disease and musculoskeletal diseases have a poor sleep in our study. A prospective cohort study found that both short sleep duration and poor sleep quality increase the risk of coronary heart disease [21]. And a meta-analysis of prospective cohort studies reported that sleep less than 7 hours or more than 8 hours are both associated with a higher risk of mortality and cardiovascular events, longer sleep may have a strong association with adverse outcomes compared with shorter sleep [22]. In another way, heart disease may affect sleep quality through biological mechanism. A crosssectional study from the Maintenance of Balance, Independent Living, Intellect, and Zest in the Elderly Boston Study found that musculoskeletal pain is significant associated with difficulties in initiating sleep, maintaining sleep or sleeping longer than usual in older adults [23]. Participants sleep poorer when they 
are more concerned about their own health, but sleep better if they reported a better health status. One reason may be that healthy people have a better sleep and they are less concerned about their own health. With the increase in frequency of alcohol drinking, participants have a poorer sleep. A study in greater Los Angeles area recruited a number of non-treatment-seeking problem drinkers showed that alcohol problem severity have a significant association with sleep disturbance [24]. Participants who have breakfast regularly have a better sleep. A cross-sectional study from the Project Eating and Activity in Teens and Young Adults study found that poor sleep quality was associated with breakfast skipping and other problematic eating behaviors [25]. Another study supported this view that breakfast consumption may improve perceived sleep quality compared with breakfast skipping in healthy young adults [26]. One possible reason may be Night Eating Syndrome, night eating may has a negative effect on sleep quality and decrease appetite in the morning. We found that people have a poorer sleep for those having a longer daily screen time. A cross-sectional study reported similar result that over use of mobile cell-phones is associated with poor sleep quality and quantity [27]. There are some possible mechanisms as follows. The first is that people take sleep time to use electronic devices [28]. Second, psychological and physical arousal due to the content of the media and social interaction may also interfere with the ability to fall and stay asleep [28]. And finally, there is the effect of light on both circadian rhythm and alertness [28].

The study results can be applied to the eligible population but may not be applied to the source population because we excluded participants which couldn't read the questionnaire or aged less than 18 or more than 90 . The study results may be applied to population from other areas which meet the eligibility criteria.

There are some limitations in our study. Firstly, this is a cross-sectional study, so causal inference was limited. Secondly, we use self-reported sleep quality rather than objective indicators. And participants' mental illness status was not investigated, which would also be associated with sleep quality. In addition, previous research has shown that there was a significant interaction between marital quality and age when studying their relationship to sleep [18]. But we weren't considering this interaction or any other interactions in our analysis. Our study didn't investigate the intake of coffee, tea or energy drink, which also could influence people's sleep quality. Future research can focus on the causal relationships and internal mechanisms between sleep quality and its associated factors.

\section{Conclusions}

In this study, we found that self-reported sleep quality was generally good in Shaanxi, China. And sleep quality was associated with gender, marital status, educational level, family monthly income, heart disease, musculoskeletal diseases, degree of concerning about their own health, self-reported health status, alcohol drinking, having breakfast, take hypnotics and daily screen time.

\section{Abbreviations}


SD: standard deviation; OR: odds ratio; cOR: crude odds ratio; aOR: adjusted odds ratio

Cl: confidence interval; BMI: body mass index.

\section{Declarations}

\section{Ethics approval and consent to participate}

The study was approved by the Ethics Committee for Medical Research, School of Public Health, Fudan University. Written informed consent was obtained from all participants or their legal guardians before investigation.

\section{Consent for publication}

Not applicable.

\section{Availability of data and materials}

The datasets are available on reasonable request. If anyone wants to get access to the data, please send an email to joycezhangyx@163.com.

\section{Competing interests}

The authors declare that they have no competing interests.

\section{Funding}

There's no fund supporting this study.

\section{Authors' contributions}

JW and $J Y$ designed the study. $Y Z, X L, B C$, and RW conducted the study. YZ performed data analysis. $Y Z$ wrote this manuscript. All the authors read, revised and approved the final manuscript.

\section{Acknowledgements}

We would like to thank the participants in this study first. We also appreciated the support of the health workers of Health Publicity and Education Center of Shaanxi province and local health workers in each city we conducted this investigation.

\section{References}


1. Cullen T, Thomas G, Wadley AJ, Myers T. The effects of a single night of complete and partial sleep deprivation on physical and cognitive performance: A Bayesian analysis. Journal of sports sciences. 2019;37(23):2726-34.

2. Javaheri S, Barbe F, Campos-Rodriguez F, Dempsey JA, Khayat R, Javaheri S, Malhotra A, MartinezGarcia MA, Mehra R, Pack Al, et al. Sleep Apnea: Types, Mechanisms, and Clinical Cardiovascular Consequences. J Am Coll Cardiol. 2017;69(7):841-58.

3. St-Onge MP. Sleep-obesity relation: underlying mechanisms and consequences for treatment. Obes Rev. 2017;18(Suppl 1):34-9.

4. Steiger A, Pawlowski M. Depression and Sleep. Int J Mol Sci. 2019;20(3):607.

5. Shan Z, Ma H, Xie M, Yan P, Guo Y, Bao W, Rong Y, Jackson CL, Hu FB, Liu L. Sleep duration and risk of type 2 diabetes: a meta-analysis of prospective studies. Diabetes Care. 2015;38(3):529-37.

6. McMakin DL, Alfano CA. Sleep and anxiety in late childhood and early adolescence. Curr Opin Psychiatry. 2015;28(6):483-9.

7. Lo K, Keung V, Cheung C, Tam W, Lee A. Associations between Sleep Pattern and Quality and Cardiovascular Risk Factors among Macao School Students. Child Obes. 2019;15(6):387-96.

8. Wang L, Qin P, Zhao Y, Duan S, Zhang Q, Liu Y, Hu Y, Sun J. Prevalence and risk factors of poor sleep quality among Inner Mongolia Medical University students: A cross-sectional survey. Psychiatry Res. 2016;244:243-8.

9. Dong X, Wang Y, Chen Y, Wang X, Zhu J, Wang N, Jiang Q, Fu C. Poor sleep quality and influencing factors among rural adults in Deqing, China. Sleep Breath. 2018;22(4):1213-20.

10. Wang Y, Li Y, Liu X, Liu R, Mao Z, Tu R, Zhang H, Zhang X, Qian X, Jiang J, et al. Gender-specific prevalence of poor sleep quality and related factors in a Chinese rural population: the Henan Rural Cohort Study. Sleep Med. 2019;54:134-41.

11. Wu W, Wang W, Dong Z, Xie Y, Gu Y, Zhang Y, Li M, Tan X. Sleep Quality and Its Associated Factors among Low-Income Adults in a Rural Area of China: A Population-Based Study. Int J Environ Res Public Health. 2018;15(9):2055.

12. Wang P, Song L, Wang K, Han X, Cong L, Wang Y, Zhang L, Yan Z, Tang S, Du Y. Prevalence and associated factors of poor sleep quality among Chinese older adults living in a rural area: a population-based study. Aging Clin Exp Res. 2019. 10.1007/s40520-40019-01171-40520.

13. Li J, Yao Y-s, Dong Q, Dong Y-h, Liu J-j, Yang L-s, Huang F. Characterization and factors associated with sleep quality among rural elderly in China. Arch Gerontol Geriatr. 2013;56(1):237-43.

14. China Wgooi. Guidelines for Prevention and Control of Overweight and Obesity in Chinese Adults. ACTA NUTRIMENTA SINICA. 2004;26(1):1-4.

15. Association AP. Diagnostic and Statistical Manual of Mental Disorders, DSM-5. Washington, DC: American Psychiatric Publishing; 2013.

16. Shim J, Kang SW. Behavioral Factors Related to Sleep Quality and Duration in Adults. J Lifestyle Med. 2017;7(1):18-26. 
17. Grenier S, Payette M-C, Gunther B, Askari S, Desjardins FF, Raymond B, Berbiche D. Association of age and gender with anxiety disorders in older adults: A systematic review and meta-analysis. Int $\mathrm{J}$ Geriatr Psychiatry. 2019;34(3):397-407.

18. Yang H-C, Suh S, Kim H, Cho ER, Lee SK, Shin C. Testing bidirectional relationships between marital quality and sleep disturbances: a 4-year follow-up study in a Korean cohort. J Psychosom Res. 2013;74(5):401-6.

19. Fok M, Stewart R, Besset A, Ritchie K, Prince M. Incidence and persistence of sleep complaints in a community older population. Int J Geriatr Psychiatry. 2010;25(1):37-45.

20. Gureje O, Oladeji BD, Abiona T, Makanjuola V, Esan O. The natural history of insomnia in the Ibadan study of ageing. Sleep. 2011;34(7):965-73.

21. Lao XQ, Liu X, Deng H-B, Chan T-C, Ho KF, Wang F, Vermeulen R, Tam T, Wong MCS, Tse LA, et al. Sleep Quality, Sleep Duration, and the Risk of Coronary Heart Disease: A Prospective Cohort Study With 60,586 Adults. J Clin Sleep Med. 2018;14(1):109-17.

22. Kwok CS, Kontopantelis E, Kuligowski G, Gray M, Muhyaldeen A, Gale CP, Peat GM, Cleator J, ChewGraham C, Loke YK, et al. Self-Reported Sleep Duration and Quality and Cardiovascular Disease and Mortality: A Dose-Response Meta-Analysis. J Am Heart Assoc. 2018;7(15):e008552-2.

23. Chen Q, Hayman LL, Shmerling RH, Bean JF, Leveille SG. Characteristics of chronic pain associated with sleep difficulty in older adults: the Maintenance of Balance, Independent Living, Intellect, and Zest in the Elderly (MOBILIZE) Boston study. J Am Geriatr Soc. 2011;59(8):1385-92.

24. Hartwell EE, Bujarski S, Glasner-Edwards S, Ray LA. The Association of Alcohol Severity and Sleep Quality in Problem Drinkers. Alcohol Alcohol. 2015;50(5):536-41.

25. Ogilvie RP, Lutsey PL, Widome R, Laska MN, Larson N, Neumark-Sztainer D. Sleep indices and eating behaviours in young adults: findings from Project EAT. Public Health Nutr. 2018;21(4):689-701.

26. Gwin JA, Leidy HJ. Breakfast Consumption Augments Appetite, Eating Behavior, and Exploratory Markers of Sleep Quality Compared with Skipping Breakfast in Healthy Young Adults. Curr Dev Nutr. 2018;2(11):nzy074-4.

27. Mohammadbeigi A, Absari R, Valizadeh F, Saadati M, Sharifimoghadam S, Ahmadi A, Mokhtari M, Ansari H. Sleep Quality in Medical Students; the Impact of Over-Use of Mobile Cell-Phone and Social Networks. J Res Health Sci. 2016;16(1):46-50.

28. Hale L, Guan S. Screen time and sleep among school-aged children and adolescents: a systematic literature review. Sleep Med Rev. 2015;21:50-8.

\section{Supplementary Files}

This is a list of supplementary files associated with this preprint. Click to download.

- QuestionnaireEnglish.docx 